\section{Genetics and Epigenetics of Hydatidiform Moles}

\author{
Rima Slim, Department of Human Genetics, McGill University Health Center \\ Research Institute, Montréal, Québec, Canada
}

Yassemine Khawajkie, Division of Experimental Medicine, McGill University Health Center, Montréal, Québec, Canada

Kurosh Rahimi, Department of Pathology, Hôpital Notre-Dame, Université de Montréal, Montréal, Québec, Canada

Philippe Sauthier, Department of Obstetrics and Gynecology, Centre Hospitalier de I'Université de Montréal (CHUM), Registre des Maladies Trophoblastiques du Québec, Montréal, Québec, Canada

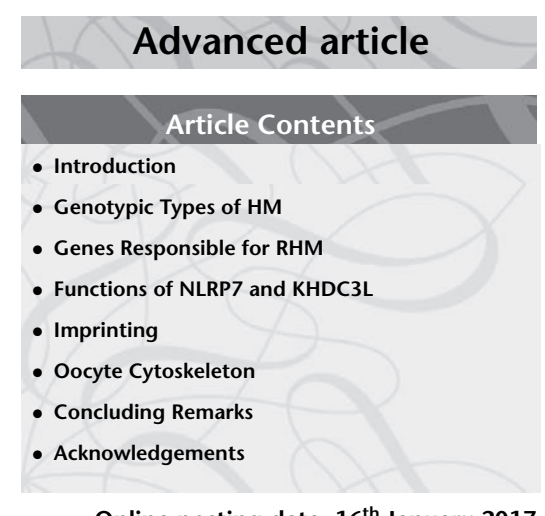

Online posting date: $16^{\text {th }}$ January 2017

\begin{abstract}
A hydatidiform mole (HM) is an abnormal human pregnancy characterised by absence of, or abnormal, embryonic development, excessive trophoblastic proliferation and hydropic degeneration of placental villi. The common types of moles are sporadic, not recurrent, and affect 1 in 1000 pregnancies in western countries. HM may recur in the same patient, which is referred to as recurrent HM (RHM), and indicates that the patient is genetically susceptible to HM. Through the examination of rare familial cases of RHM, two maternal-effect genes, NLRP7 and KHDC3L, responsible for this condition have been identified. Pathogenic variants in these genes appear to impair imprinting establishment during oogenesis. Herein, we review current knowledge on the genetics and epigenetics of RHM, and highlight the benefits of testing patients for pathogenic variants in the known genes.
\end{abstract}

\section{Introduction}

The clinical manifestations of hydatidiform mole (HM) have greatly evolved throughout the years. The first clinical descriptions were made by Hippocrates in 400 BC who described the presence of intrauterine vesicles. It was only in 1276 that a more 'precise' clinical description appeared. At that time, according to the medieval legend of Countess Margaret of Henneberg, who

eLS subject area: Genetics \& Disease

How to cite:

Slim, Rima; Khawajkie, Yassemine; Rahimi, Kurosh; and

Sauthier, Philippe (January 2017) Genetics and Epigenetics of

Hydatidiform Moles. In: eLS. John Wiley \& Sons, Ltd: Chichester.

DOI: 10.1002/9780470015902.a0026452 had most probably spontaneously ejected an HM, the vesicles were believed to be the immature birth of ' 365 children' (Bondeson and Molenkamp, 1996). Unfortunately, the Countess died the next day of heavy bleeding. This surely is the first description of a spontaneous evolution of a complicated HM.

The clinical manifestation of moles by the spontaneous ejection of vesicles, heavy bleeding and uterine rupture is still seen nowadays in developing countries where medical follow-up of pregnancies does not start at the eighth week of gestation, and consequently HM can freely evolve. However, in developed countries, the current clinical signs are usually vaginal bleeding, sensation of heaviness in the pelvic region, enlargement of the uterus and excessive nausea and vomiting (hyperemesis). Because these symptoms are frequent in the first stages of normal pregnancies, most clinicians discover moles by ultrasound performed on cases of abnormal evolution of a pregnancy in the first 8 weeks of gestation or at the first gynaecological visit (between 6 and 8 weeks) (Hou et al., 2008; Mangili et al., 2008; Sebire et al., 2001).

\section{Clinical presentation}

An HM is suspected when ultrasonography reveals echogenic structures all over the uterus and this is referred to as a "snow storm' with the absence of a defined gestational sac or with the presence of a gestational sac as well as some embryonic structures. Uncommon clinical manifestations can develop in advanced diseases such as hyperthyroidism when human chorionic gonadotropin (hCG) is above $100000 \mathrm{U} \mathrm{mL}^{-1}$ (Amir et al., 1984). Luteinising cysts of the ovarian theca cells may also be seen as a result of ovarian hyperstimulation due to the rise in hCG. In the majority of cases, the cysts will disappear when the mole is removed. However, in rare cases, the cysts may twist and induce violent abdominal pain (Osathanondh et al., 1986). Preeclampsia has also been described around 20 weeks of gestation in some patients with HM, but this clinical manifestation is rare in developed countries (Ramsey et al., 1998).

The clinical evaluation of a patient with a suspected HM by ultrasound will include a detailed history of all systems, in particular those related to obstetrics and gynaecology. A pelvic medical 
examination will show an enlarged uterus and eventually the presence of cysts in the adnexaes. This initial suspicion of the presence of an HM is followed by a laboratory evaluation of the level of hCG, which is higher in molar than in normal pregnancies of a similar gestational stage; an assessment of thyroid functions and an X-ray of the lungs to search for embolus moles or pleural effusions (accumulation of fluid around the lung). Abnormal values for these tests, combined with the ultrasound result, are in favour, but not diagnostic, of an HM. However, when the pregnancy is not viable, the clinician will perform dilatation and curettage suction of the product of conception. This product of conception will then be sent for histopathological examination, based on which the final diagnosis is established.

\section{Epidemiology}

$\mathrm{HM}$ is a disease that displays a wide geographic distribution in its frequencies with a gradient of increasing frequencies from West to East. The highest frequencies reach 1 in 60 pregnancies and are found in Southeastern Asia such as in Indonesia and the Philippines, and the lowest frequencies, 1 in 1000 to 1 in 1500 pregnancies, are found in the United States, Canada and Europe (Grimes, 1984). These frequencies mostly represent the common sporadic HM as recurrent hydatidiform moles (RHMs), defined by the occurrence of at least two HM in the same patient, are rare and account for only $1.4-9.4 \%$ of all HM. Similar to sporadic HM, RHM have been reported to be more frequent in Morocco (9.4\% of all HM) (Boufettal et al., 2011), Lebanon (6\%) (Kronfol et al., 1969), and Korea (4.3\%) (Kim et al., 1998). The lowest frequencies of RHM have been reported in the United States (1.4-1.5\%) (Berkowitz et al., 1998) and Europe (1.8\%) (Sebire et al., 2003).

\section{Histopathology}

The classical macroscopic features (visible to the naked eyes) of a typical HM used to include severe oedematous and hydropic chorionic villi (Figure 1) that have a grape-like appearance. However, because of the standard use of ultrasonography in current medical practice, most HM are diagnosed and evacuated at earlier stages, before the excessive hydropic degeneration of the chorionic villi and their characteristic grape-like appearance. Consequently, the obvious manifestation of HM by macroscopy, as shown in Figure 1, is becoming much less frequent. Evacuated products of conception are sent to histopathology laboratories where they are fixed and embedded into paraffin blocks. The tissues are then sectioned, mounted on microscopic slides, stained with hematoxilin and eosin, and examined by light microscopy by pathologists. Microscopic examination of a product of conception clinically suspected to be an HM has two goals - the first is to determine whether the product of conception corresponds to an HM or a nonmolar arrested pregnancy and the second is to classify the HM into two histopathological types, complete hydatidiform mole (CHM) and partial hydatidiform mole (PHM), because these two types have different risks of malignant degeneration into neoplasias.

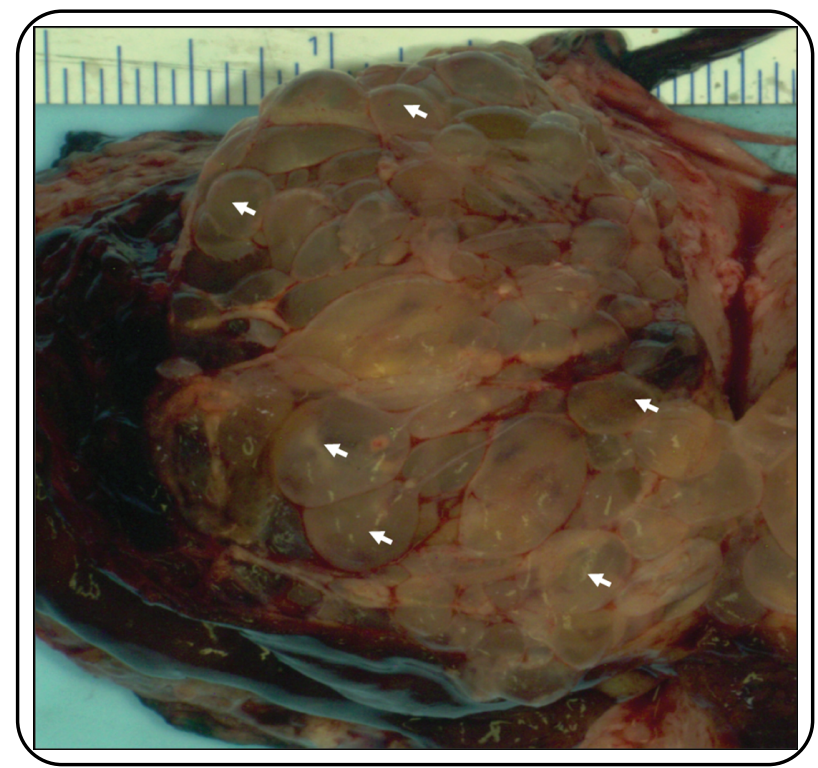

Figure 1 Gross morphology of a hydatidiform mole. The photo was taken directly after the evacuation of the HM by curettage and suction. Arrows indicate some vesicles.

\section{Complete hydatidiform mole}

On the basis of the histopathological features, HM are divided into CHM and PHM. Microscopic features of CHM consist of clubbing of the chorionic villi, the presence of apoptotic bodies referred to as karyorrhexis, uniformity in the shape of chorionic villi, stromal hypercellularity and nonpolarised, circumferential trophoblastic hyperplasia with atypia (cells with abnormal nuclear morphology) (Figure 2a) (Szulman and Surti, 1978). In general, all the chorionic villi display oedema and cisterns with unpolarised and haphazard trophoblastic proliferation. The rim of stroma at the periphery of chorionic villi is often rich in cells admixed with apoptotic bodies (Figure $\mathbf{2 b}$ ). Trophoblastic inclusions may be seen but are not a common feature of CHM. Exaggerated placental site is frequently associated with CHM but rarely with PHM. The presence of foetal tissues or nucleated red blood cells inside the chorionic villi excludes the diagnosis of $\mathrm{CHM}$, except in rare cases of twin pregnancies (consisting of a foetus and a mole), mosaicism and chimerism. In conclusion, the specific features of CHM are club-shaped chorionic villi, oedematous chorionic villi with cistern, karyorrhexis and excessive circumferential trophoblastic proliferation.

\section{Partial hydatidiform mole}

PHM macroscopically displays two populations of oedematous and small fibrotic chorionic villi (Figure 2c). Upon microscopic evaluation, apoptotic bodies are usually absent but might be seen focally in some cases. Cisterns could be present in some chorionic villi, but they are not as frequent as in CHM. Excessive circumferential trophoblastic proliferation and atypia are absent in PHM. Extraembryonic tissues (chorion and amnion, which constitute the foetal membranes) (Figure 2d) and/or embryonic tissues such 


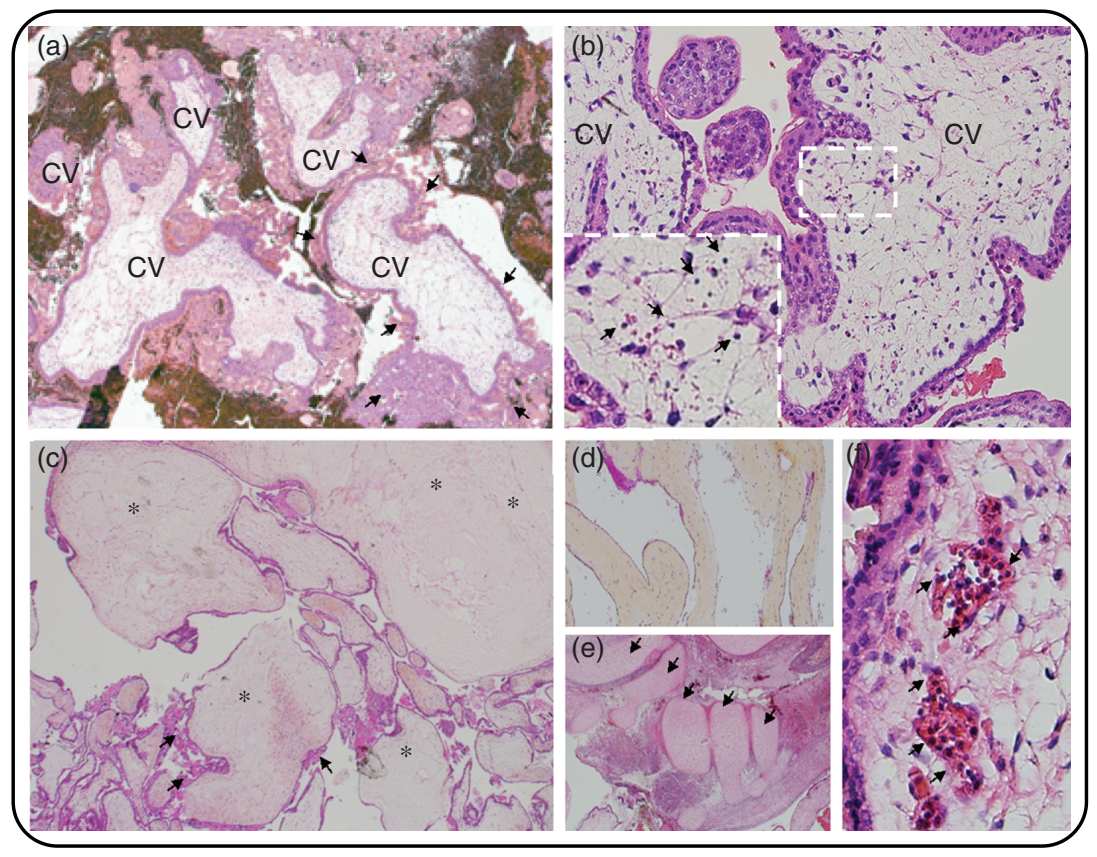

Figure 2 Histopathology of complete and partial hydatidiform moles. (a) A microphotograph of a CHM. CV stands for chorionic villi and arrows indicate circumferential trophoblastic proliferation around one chorionic villous. (b) Two chorionic villi displaying important karyorrhexis. The inset shows a higher magnification of karyorrhectic debris (arrows). (c) A microphotograph of a partial hydatidiform mole showing the presence of two populations of chorionic villi, the large hydropic chorionic villi (asterisk) and the small ones. Focal trophoblastic proliferation around one chorionic villous is indicated by arrows. A microphotograph of a different PHM showing foetal membranes in (d) and some skeletal bones of embryonic origin (arrows) in (e). (f) A microphotograph of a PHM showing nucleated red blood cells (arrows) inside a chorionic villous.

as skeletal bones (Figure 2e), cartilages and nucleated foetal red blood cells inside the chorionic villi may be present (Figure 2f) (Szulman and Surti, 1978). In addition, a complete foetus with normal or abnormal morphology may be present in PHM. In conclusion, the most characteristic features of PHM include the presence of two populations of chorionic villi, irregular contour of chorionic villi and moderate trophoblastic proliferation around the chorionic villi.

\section{Genotypic Types of HM}

\section{Methods}

The methods used to determine the genotypes of HM and the parental contribution to their genomes have evolved with time from karyotype analysis by classical culture-based cytogenetics to a wide range of DNA (deoxyribonucleic acid)-based methods that include flow cytometry, fluorescent in situ hybridisation with probes against various centromeric or heterochromatin repeats, and multiplex and/or simplex microsatellite genotyping. Another method, p57 ${ }^{\mathrm{KIP} 2}$ immunohistochemistry, introduced in 2001 (Castrillon et al., 2001), has become an important one and is being performed on a routine basis in many laboratories as part of $\mathrm{HM}$ assessments. This method is currently used to identify, indirectly, the presence of the maternal genome in molar tissues. P57 ${ }^{\mathrm{KIP} 2}$ is the protein coded by cyclin-dependent kinase inhibitor $1 \mathrm{C}, C D K N 1 C$, which is paternally imprinted and expressed only from the maternal genome in the nuclei of normal first trimester cytotrophoblastic and villous stroma cells (Castrillon et al., 2001). Therefore, the presence of nuclear staining for $\mathrm{p} 57^{\mathrm{KIP} 2}$ in these cells indicates that these cells contain at least one copy of the maternal genome. The advantage of $\mathrm{p} 57^{\mathrm{KIP} 2}$ immunohistochemistry is that it is simple, inexpensive and can be performed on sections of formalin-fixed paraffin embedded (FFPE) tissues that are prepared systematically from all molar pregnancies as part of patient care.

The other DNA-based methods used to be performed only on fresh tissues. However, nowadays, all can be performed on FFPE tissues. Importantly though, one needs to keep in mind that working with FFPE tissues is technically more challenging than fresh tissues. Therefore, when genotyping FFPE tissues, it is advisable to use more than one method to minimise mistakes due to technical difficulties caused by the low quality of DNA from archived FFPE tissues and the difficulty in some cases in isolating chorionic villi and separating them from maternal endometrial cells. From our experience, we find that using several methods and reconciling their data allow us, in addition to minimising potential mistakes, to confirm the genotypes of unusual HM cases. This will consequently improve our understanding of this pathology by drawing better and more accurate correlations between the genotype of the product of conception and their histopathological features. 
Table 1 Summary of the main genotypic types of sporadic and recurrent HM

\begin{tabular}{lll}
\hline & CHM & PHM \\
\hline Sporadic HM & Diploid androgenetic & monospermic, $\mathbf{X X}$ \\
& Diploid androgenetic dispermic, & Triploid dispermic XXX, XXY \\
and XYY & \\
& $\mathbf{X X}$ or $\mathbf{X Y}$ & \\
& Tetraploid $4 \mathrm{n}, \mathbf{X X Y Y}, \mathbf{X X X X}$, & \\
& $\mathbf{X X X X}, \mathbf{X X X Y}, \mathbf{X Y Y} \mathbf{Y}$ & \\
& Diploid biparental & \\
& Diploid biparental, $\mathbf{X X}$ or $\mathbf{X Y}$ & \\
$\begin{array}{l}\text { RHM from patients with } \\
\text { recessive pathogenic variants } \\
\text { in } N L R P 7 \text { or } K H D C 3 L\end{array}$ & & \\
\hline
\end{tabular}

Pink and blue colours are used to indicate the maternal and paternal genetic complements.

${ }^{a}$ The genotypes of these HM need to be revisited based on emerging data in the field in order to validate their existence.

\section{Genotypic types of HM}

\section{Diploid androgenetic}

The use of the various methods mentioned above has shown that common sporadic nonrecurrent CHMs are mostly androgenetic. In the majority of the cases (80-90\%), they are monospermic and contain two copies of a single paternal set of 23 chromosomes originating from one spermatozoid (androgenetic monospermic). Such HM are always XX because YY zygotes or embryos are not viable (Table 1). In up to $20 \%$ of the cases, CHMs contain two paternal sets of chromosomes deriving from two different spermatozoids. These HM are androgenetic dispermic and can be XX or XY.

\section{Triploid dispermic}

Sporadic, nonrecurrent PHMs are mostly triploid with 69 chromosomes consisting of two sets of paternal chromosomes originating from two different spermatozoids and one set of maternal chromosomes. These HM are said to be triploid dispermic and can be XXX, XXY or XYY (Table 1).

\section{Unusual and rare genotypes}

Other rare genotypic types of HM that have, or mimic, the morphology of CHM or PHM have also been reported. These include tetraploid HM, aneuploid diploid biparental HM and diploid biparental HM with no aneuploidies (Table 1). Such $\mathrm{HM}$ represent a small fraction of all HM and therefore will not be discussed in this article with the exception of RHM from patients with inherited recessive pathogenic variants in NLRP7 or $K H D C 3 L$. These RHM have diploid biparental genome with no detectable aneuploidies and are discussed below.

\section{Genes Responsible for RHM}

\section{NLRP7}

NLRP7 maps to 19q13.4 (Moglabey et al., 1999) and was the first maternal-effect gene to be identified in humans (Murdoch et al., 2006). Maternal-effect genes are a subset of genes that are needed in the oocytes, in the form of RNA (ribonucleic acid) or proteins, to sustain normal embryonic development until the activation of the embryonic genome at both the transcriptional and translational levels. NLRP7 codes for a protein that is a member of the NOD-like receptor pyrin-containing domain 7 . It has three main domains: a pyrin domain; a NACHT domain, which contains an ATPase (adenosine triphosphatase) domain, and leucine-rich repeat domains formed by 9-10 repeats, depending on splicing isoforms (Figure 3). The causal role of NLRP7 in the aetiology of RHM was reported in 2006 by our group based on the identification of recessive pathogenic variants in four unrelated patients from different populations (Murdoch et al., 2006). To date, approximately 64 different pathogenic variants in NLRP7 have been seen in a recessive state and in a total of approximately 150 patients (Figure 3) (http://fmf.igh.cnrs.fr/ISSAID/infevers/). Available reports on large cohorts indicate that recessive pathogenic variants in $N L R P 7$ are not present at the same frequency in all populations; while China seems to have the lowest frequency (58\%) (Qian et al., 2011), Pakistan (Hayward et al., 2009), India (Slim et al., 2009) and Mexico (Estrada et al., 2013) have the highest frequencies ranging from $81 \%$ to $85 \%$. Because $N L R P 7$ is a maternal-effect gene and the primary defect in patients with two defective alleles is in their oocytes, three patients have so far tried donated ova after in vitro fertilisation and had successful pregnancies leading to healthy children (Akoury et al., 2015a; Fisher et al., 2011). The benefit of ovum donation for patients with RHM and recessive pathogenic variants in NLRP7 highlights the importance of offering DNA testing for these patients. Indeed, such a situation is rare in reproductive medicine where we can know the exact defect at the nucleotide level and offer the appropriate assisted reproductive technology service for the patients.

\section{KHDC3L}

The analysis of patients with RHM who were negative for pathogenic variants in NLRP7 led to the identification of a second maternal-effect gene, KHDC3L, for this condition (Parry et al., 2011). KHDC3L maps to human $6 \mathrm{q} 13$ and is a small protein of 


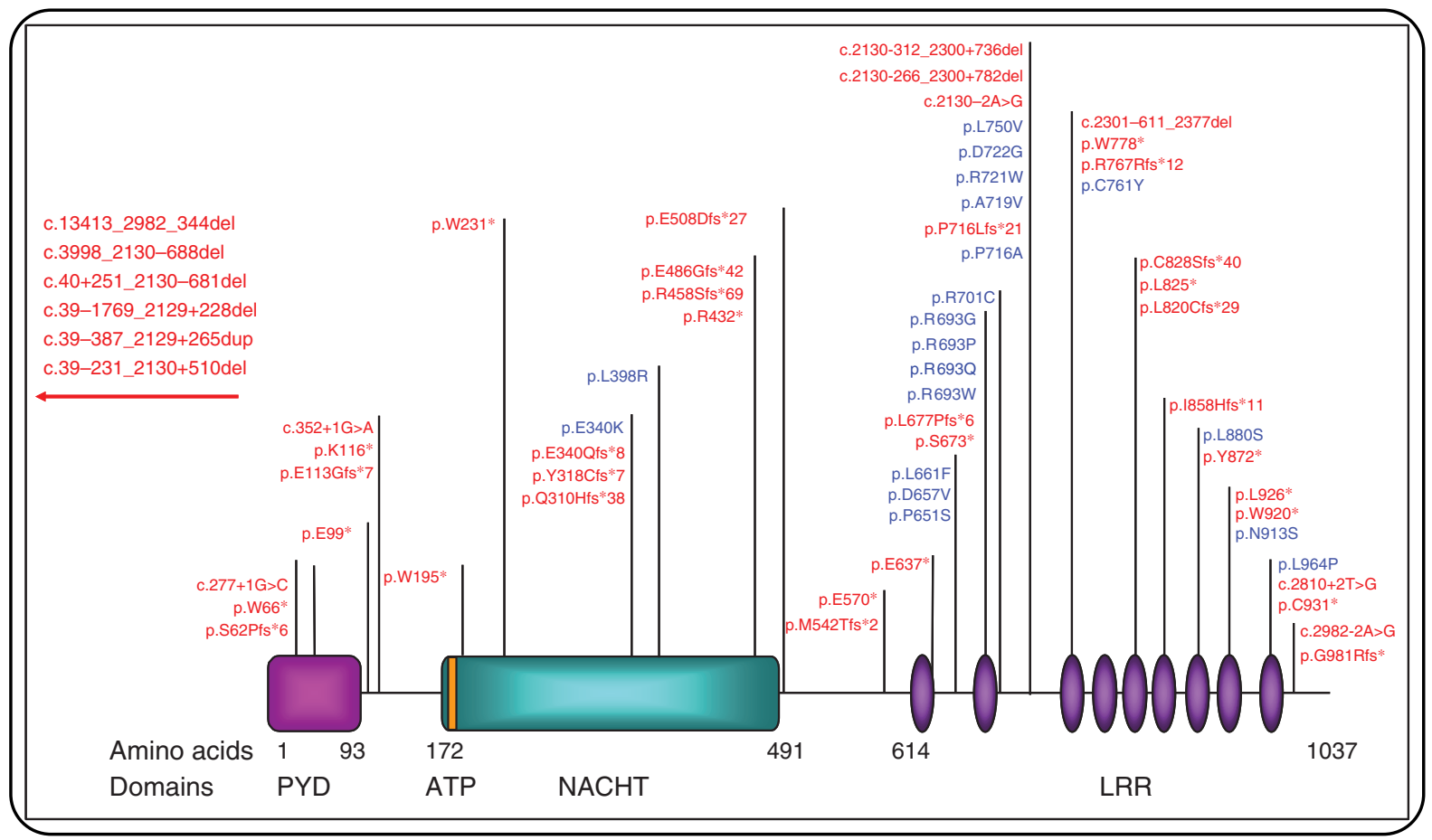

Figure 3 NLRP7 protein structure and reported pathogenic variants observed in a recessive state by various groups (http://fmf.igh.cnrs.fr/ISSAID/infevers/). NLRP7 protein has mainly three domains. Protein truncating variants (stop codon, deletions, insertions and invariant splice mutations) are in red and missense variants are in blue. The large deletions that begin before the start codon are indicated by an arrow towards the $5^{\prime}$ untranslated region.

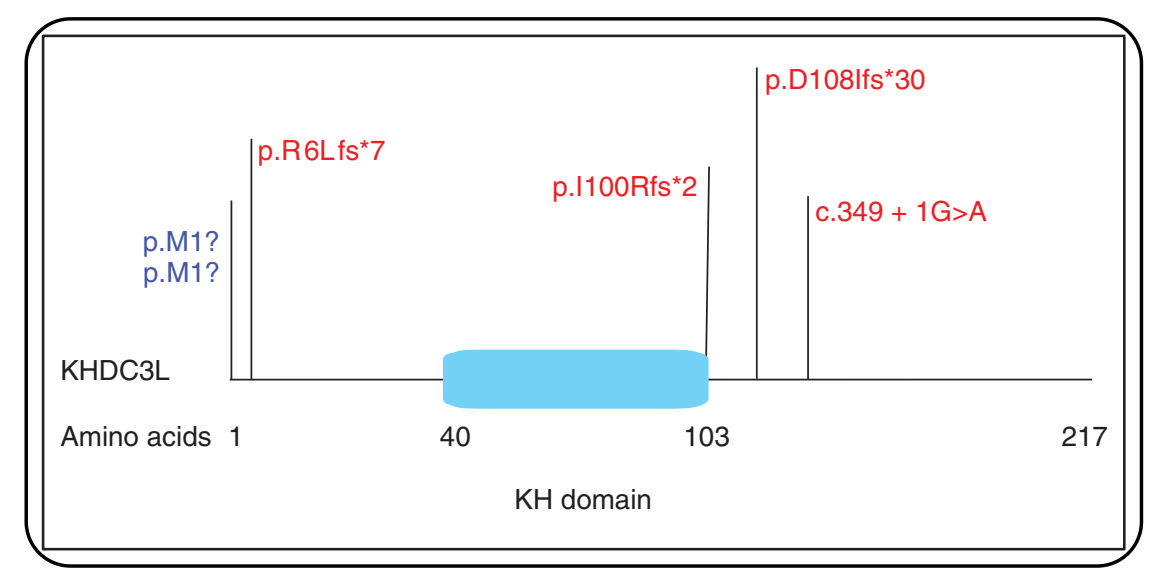

Figure $4 \mathrm{KHDC} 3 \mathrm{~L}$ protein structure and reported pathogenic variants in recessive state by various groups. The two pathogenic variants affecting the start codon are indicated by question marks because their consequences on the protein are not known (http://databases.lovd.nl/shared/genes/KHDC3L).

217 amino acids. It contains an atypical KH domain. To date, six different pathogenic variants in $K H D C 3 L$ have been reported in a total of 10 patients (Figure 4) (Parry et al., 2011; Reddy et al., 2013; Rezaei et al., 2016). To our knowledge, none of the reported patients with pathogenic variants in $K H D C 3 L$ have tried in vitro fertilisation with donated ova. However, by analogy to NLRP7, we expect that this assisted reproductive technology would rescue the defects in these patients.

\section{Functions of NLRP7 and KHDC3L}

\section{RHM genotypes}

The existence of RHM was described a long time ago, but it was only in recent years that RHM cases were characterised at the genomic DNA level. The parental contribution to the first case of RHM was described by in 1991 by Vejerslev et al. (1991) and 
Table 2 Recapitulation of HM genotypes from patients with recessive pathogenic variants in $N L R P 7$ or $K H D C 3 L$

\begin{tabular}{llll}
\hline & Diploid biparental & Triploid dispermic & Triploid digynic \\
\hline NLRP7 & $118(98 \%)$ & $1(0.008 \%)$ & $1(0.008 \%)$ \\
KHDC3L & $9(90 \%)$ & & $1(10 \%)$ \\
\hline
\end{tabular}

these RHM were found diploid biparental with a normal parental contribution to the molar genomes as opposed to sporadic CHM or PHM. This RHM case, in addition to others from familial and nonfamilial cases, contributed to the identification of NLRP7 and KHDC $3 L$.

Now, a decade after the identification of $N L R P 7$, the parental contribution to approximately $118 \mathrm{HM}$ tissues from patients with recessive pathogenic variants in NLRP7 have been reported. All these tissues were found diploid biparental with the exception of two tissues that were found triploid, one dispermic (Ulker et al., 2013) and one digynic (Fallahian et al., 2013) (Table 2). For $K H D C 3 L$, the parental contribution to only $10 \mathrm{HM}$ from patients with recessive pathogenic variants has been reported. Nine of these tissues were found diploid biparental (Fallahian et al., 2013; Hayward et al., 2009; Judson et al., 2002; Reddy et al., 2013) and one triploid digynic (Fallahian et al., 2013) (Table 2). Despite their diploid biparental genome, at the histopathological level, RHM caused by pathogenic variants in NLRP7 or KHDC $3 L$ mimic the sporadic HM and some are diagnosed by histopathology as CHM (Helwani et al., 1999; Messaed et al., 2011b; Sebire et al., 2013; Zhao et al., 2006), PHM (Helwani et al., 1999; Vejerslev et al., 1991), or atypical HM (Sebire et al., 2013). Studies on two large cohorts of RHM agree on the facts that HM from patients with inherited defects in either gene have, in general, less trophoblastic proliferation than androgenetic CHM (Messaed et al., 2011b; Sebire et al., 2013).

Not all RHM are diploid biparental, some are androgenetic monospermic (Dixon et al., 2012; Eagles et al., 2016) and others are triploid dispermic (Eagles et al., 2016; Slim et al., 2011). However, highly RHM are mostly diploid biparental and most are from patients with recessive pathogenic variants in NLRP7 and few from patients with recessive pathogenic variants in $K H D C 3 L$.

\section{Imprinting}

Before the identification of NLRP7 as a causative gene for RHM, the demonstration that RHM are diploid biparental and are morphologically similar to diploid androgenetic HM raised the hypothesis about the potential role of wild-type NLRP7 in establishing imprinting marks during oogenesis or maintaining them during early embryonic development (Sunde et al., 1993). This interesting hypothesis was at the origin of several studies that assessed DNA methylation of several differentially methylated region (DMR) associated with imprinted genes in diploid biparental HM from patients with pathogenic variants in $N R L P 7$ or $K H D C 3 L$. The results were as expected for imprinted, maternally methylated DMRs, and these DMRs were found to lack their methylation marks, therefore mimicking their corresponding paternal DMR. This finding was originally documented by Judson et al. (2002) and was since then replicated in every analysed tissue from patients with recessive pathogenic variants in NLRP7 or KHDC3L (El-Maarri et al., 2003; Hayward et al., 2009; Ito et al., 2015; Kou et al., 2008; Sanchez-Delgado et al., 2015).

The proposed explanations of such lack of DNA methylation is that the establishment or setting of methylation imprinting marks during oogenesis did not occur because of the pathogenic variants in NLRP7 or KHDC3L. All these studies investigated the methylation at few DMR (4-9) (El-Maarri et al., 2005; Hayward et al., 2009; Ito et al., 2015; Judson et al., 2002; Kou et $a l ., 2008$ ) with the exception of a recent genome-wide DNA methylation study using the Illumina Infinium HumanMethylation450 Bead-Chip arrays (Sanchez-Delgado et al., 2015). This comprehensive and important study confirmed the lack of methylation on several maternally methylated DMR and expanded this observation to several additional DMR. The authors proposed an interesting mechanism involving a disruption in the selection and recruitment of follicles at a specific time during the foetal life of patients with pathogenic variants in NLRP7 or KHDC $3 L$ that coincides with the time of methylation mark establishment, and would result in the ovulation of oocytes with inappropriate methylation marks (Sanchez-Delgado et al., 2015). This suggestion is interesting and plausible because it was found that in mice, several Nlrp genes are under the transcriptional control of factor in the germ line, alpha (FIGLA) whose knockout leads to female mice with no primordial follicles at birth (Joshi et al., 2007). It is therefore possible that the oocyte defect in women with recessive pathogenic variants in NLRP7 or KHDC $3 \mathrm{~L}$ occurred earlier during folliculogenesis. In addition, the study by Sanchez-Delgado et al. (2015) is the only one that checked the transcriptional consequences of the lack of methylation marks and demonstrated the biallelic expression of all analysed genes. Moreover, this study confirmed a previous observation that the methylation defect in RHM from patients with recessive pathogenic variants in NLRP7 is restricted to imprinted genes (Djuric et al., 2006) and only affects the conceptions of these patients (El-Maarri et al., 2005).

In conclusion, studies from various groups are concordant with the lack of DNA methylation only on the DMR of most imprinted, maternally methylated genes, probably due to a defect during folliculogenesis or oogenesis, and as a consequence of recessive pathogenic variants in $N L R P 7$ or $K H D C 3 L$.

\section{Other roles of NLRP7 and KHDC3L}

Functional studies aimed at understanding the role of NLRP7 in the pathogenesis of RHM have been hampered by the lack of an animal model to study this disease as NLRP7 does not have orthologues in rodent or bovine. In humans, the closest gene to NLRP7 is NLRP2, which lies $25 \mathrm{~kb}$ distal to NLRP7 in a head-to-head orientation. NLRP7 and NLRP2 share a similar genomic structure and richness in Alu elements (Reddy et al., 
2016). In addition, the same richness in Alu elements is found in all their primate orthologues from chimpanzee to marmoset, indicating that these Alu elements have been inserted in the common $N L R P 2 / 7$ ancestor before its duplication into two genes. These recent observations corroborate a previous suggestion on the duplication of the common, $N L R P 2 / 7$, ancestor into two separate genes in primates (Tian et al., 2009). Because of the absence of a rodent or bovine orthologues of $N L R P 7$, functional studies to dissect its function have all been carried out on cellular models.

Regulation of inflammation. NLRP7 is a member of the NLRP protein family that consists of 14 members in humans, named NLRP1, NLRP2, NLRP3, and so on up to NLRP14. Two of these proteins, NLRP3 and NLRP12, have been shown to play a causal role in inflammatory diseases. When mutated, these genes lead to abnormal excessive activation of the inflammasome, a large multiprotein complex that results in the production of the proinflammatory cytokines IL1B and IL18. Studies from various groups and in different cellular models demonstrated that NLRP7 forms an inflammasome in response to stimulation by bacterial-derived products (acylated lipoproteins, lipospolysaccharides, etc.) (Khare et al., 2012; Singer et al., 2014; Zhou et al., 2016) and downregulates pro-IL1B secretion in stably transfected monocytic cell lines (Khare et al., 2012) as well as in peripheral blood mononuclear cells from patients with pathogenic variants (Messaed et al., 2011a). In addition, in transient transfections, overexpressing NLRP7 downregulates pro-IL1B production (Kinoshita et al., 2005; Messaed et al., 2011a).

\section{Trophoblastic differentiation and proliferation}

The role of NLRP7 in trophoblastic differentiation was first described in human $\mathrm{H} 9$ embryonic stem cells, where NLRP7 knockdown led to accelerated expression of trophoblastic differentiation markers (Mahadevan et al., 2014). The same conclusion was also reached in a different study designed by our group to look for a correlation between HM features and the nature of the pathogenic variants in the patients. We found that severe (protein-truncating) pathogenic variants in the patients are associated with the absence of embryonic tissues of inner cell mass origin in the molar conception and excessive trophoblastic proliferation (Nguyen et al., 2014). However, milder (missense) pathogenic variants were associated with the presence of some embryonic tissues and mild trophoblastic proliferation.

\section{Oocyte Cytoskeleton}

In human oocytes, where NLRP7 and KHDC3L proteins play their primary roles, the two proteins colocalise perfectly, form a cytoskeleton that is different from that of $\alpha$-tubulin, and are more abundant at the cytocortex (Akoury et al., 2015b). After fertilisation, both proteins move to the outer cortical region and are not present at the cell-to-cell contact regions. Basically, the localisation of NLRP7 and KHDC3L in human oocytes mimics that of KHDC3 (the mouse orthologue of human KHDC3L, also called FILIA) and NLRP5 in mouse oocytes and early embryos; the only difference is that human KHDC3L enters the nuclei starting from the morula stage where it remains until the blastocyst stage (Akoury et al., 2015b).

The localisation of NLRP7 and KHDC3L to the oocyte cytoskeleton raises an important question about how these cytoskeletal proteins affect trophoblast differentiation and proliferation. For sure, we do not know the answer to this question. However, if we were to speculate, one possibility is that the oocytes may have several other defects aside from the lack of DNA methylation on maternally imprinted DMR that would altogether be responsible for the lack of embryonic tissue differentiation. Another possibility is that NLRP7 and KHDC3L may be involved in intracellular trafficking of RNA and proteins that are essential for the activation of the embryonic genome. The fact that zygotes with different genotypes, diploid biparental, diploid androgenetic monospermic, diploid androgenetic dispermic and triploid dispermic genomes lead to HM suggests that the decision to develop into an HM is taken before the activation of the embryonic genome, which does not seem to be making a big difference.

\section{Roles of KHDC3L}

To date, no functional studies have been performed to address the role of KHDC3L protein in human cells. In mice, KHDC3 colocalises and interacts with NLRP5 in the oocyte cortical region, where the protein stability of FILIA depends on the presence of NLRP5 (Ohsugi et al., 2008). In another study on mice, null females for $\mathrm{Khdc}^{-/-}$were found to have decreased fecundity due to abnormal spindle formation, chromosome misalignment in embryos, failure of the spindle assembly checkpoint and defective RhoA signalling (Zheng and Dean, 2009), which can be explained by an abnormal oocyte cytoskeleton.

\section{Concluding Remarks}

Studying rare Mendelian forms of common diseases is an opportunity to easily identify their causative genes, dissect the functions of their proteins and better understand the pathogenesis of the multifactorial forms of the diseases. Now, a decade after the identification of the first causative gene for RHM, what have we learned from the work on $N L R P 7$ and $K H D C 3 L$ and how does this knowledge explain the sporadic common form of HM?

At the genotypic level, RHM from patients with recessive $N L R P 7$ or $K H D C 3 L$ pathogenic variants are diploid biparental and originate from a different mechanism than sporadic androgenetic or triploid HM.

At the epigenetic level, RHM from patients with recessive pathogenic variants in $N L R P 7$ or $K H D C 3 L$ lack maternal methylation marks on maternally methylated DMR, and mimic diploid androgenetic CHM, which lack a maternal genome.

RHM from patients with recessive pathogenic variants in NLRP7 have defective oocytes and benefit from ovum donation. In human oocytes, NLRP7 and KHDC3L proteins colocalise to the cortical region. Similarly, all sporadic HM are believed to be caused by defects in the oocytes. Therefore, HM, in general, result from inherited or acquired defects affecting the oocytes. 
Diploid biparental HM from patients with pathogenic variants in NLRP7 have an imbalance between embryonic tissue differentiation and trophoblastic proliferation. This imbalance is also observed in androgenetic monospermic/dipsermic and triploid dispermic HM. The similarity between these different genotypic types of $\mathrm{HM}$ is certainly fascinating and remains to be elucidated in future studies.

$N L R P 7$ is part of the innate immune system and its pathogenic variants downregulate inflammation. Similarly, patients with sporadic $\mathrm{HM}$ have a weak cellular-mediated immunity in response to phytohemagglutinin and concanavalin A, and delayed skin hypersensitivity to dinitrochlorobenzene, purified protein derivatives, and recall Candida antigens (Ho et al., 1980; Khanna et al., 1985; Tomoda et al., 1976)

Another similarity between inherited and sporadic HM is that most patients with pathogenic variants in NLRP7 or KHDC $3 L$ originate from countries with high incidence of sporadic HM. In several of these countries, founder pathogenic variants in NLRP7 (Estrada et al., 2013; Kou et al., 2008; Slim et al., 2009) and KHDC3L (Reddy et al., 2013) have been identified. In addition, variants in NLRP7 have been shown to display a gradient of increasing frequencies from North to South (Slim et al., 2012). Our current explanation is related to NLRP7's role in the immune system, which is known to display important differences between Northern and Southern populations. We believe that NLRP7 defects are prevalent in Southern populations because they may have historically conferred some selective advantages for these populations, possibly against some infectious diseases. It is important to note that HM manifest in the first trimester of pregnancy (8-12 weeks), despite the fact that the embryos had stopped developing much earlier. Consequently, an important feature for the manifestation of any HM is its retention and delayed rejection by the maternal immune system.

In conclusion, the identification of two genes for RHM has, in general, advanced our understanding of the pathology of HM. The challenges ahead are to fully understand the roles NLRP7 and $K H D C 3 L$ and better understand the risk factors for common $\mathrm{HM}$ and how each risk factor predisposes to HM and to which genotypic type.

\section{Acknowledgements}

I am indebted to past and current members of my laboratory who worked on this disease throughout the years. I thank them for their energy, enthusiasm and discussions. I also thank Ngoc Minh Phuong Nguyen for her critical reading of the manuscript and all physicians who refer their patients to me and continue to enrich our understanding of this pathology. RS is supported by the Canadian Institute of Health Research (MOP-86546, POP-122897, and MOP-130364).

\section{References}

Akoury E, Gupta N, Bagga R, et al. (2015a) Live births in women with recurrent hydatidiform mole and two NLRP7 mutations. Reproductive Biomedicine Online 31 (1): 120-124.
Akoury E, Zhang L, Ao A and Slim R (2015b) NLRP7 and KHDC3L, the two maternal-effect proteins responsible for recurrent hydatidiform moles, co-localize to the oocyte cytoskeleton. Human Reproduction 30 (1): 159-169.

Amir SM, Osathanondh R, Berkowitz RS and Goldstein DP (1984) Human chorionic gonadotropin and thyroid function in patients with hydatidiform mole. American Journal of Obstetrics and Gynecology 150 (6): 723-728.

Berkowitz RS, Im SS, Bernstein MR and Goldstein DP (1998) Gestational trophoblastic disease. Subsequent pregnancy outcome, including repeat molar pregnancy. Journal of Reproductive Medicine 43 (1): 81-86.

Bondeson J and Molenkamp A (1996) The Countess Margaret of Henneberg and her 365 children. Journal of the Royal Society of Medicine 89 (12): 711-716.

Boufettal H, Coullin P, Mahdaoui S, et al. (2011) Complete hydatiforme mole in Morocco: epidemiological and clinical study. Journal de Gynecologie, Obstetrique et Biologie de la Reproduction 40 (5): 419-429.

Castrillon DH, Sun D, Weremowicz S, et al. (2001) Discrimination of complete hydatidiform mole from its mimics by immunohistochemistry of the paternally imprinted gene product p57KIP2. American Journal of Surgical Pathology 25 (10): 1225-1230.

Dixon PH, Trongwongsa P, Abu-Hayyah S, et al. (2012) Mutations in NLRP7 are associated with diploid biparental hydatidiform moles, but not androgenetic complete moles. Journal of Medical Genetics 49 (3): 206-211.

Djuric U, El-Maarri O, Lamb B, et al. (2006) Familial molar tissues due to mutations in the inflammatory gene, NALP7, have normal postzygotic DNA methylation. Human Genetics 120 (3): 390-395.

Eagles N, Sebire NJ, Short D, et al. (2016) Risk of recurrent molar pregnancies following complete and partial hydatidiform moles. Human Reproduction 31 (6): 1379.

El-Maarri O, Seoud M, Coullin P, et al. (2003) Maternal alleles acquiring paternal methylation patterns in biparental complete hydatidiform moles. Human Molecular Genetics 12 (12): 1405-1413.

El-Maarri O, Seoud M, Riviere JB, et al. (2005) Patients with familial biparental hydatidiform moles have normal methylation at imprinted genes. European Journal of Human Genetics 13 (4): 486-490.

Estrada H, Buentello B, Zenteno JC, Fiszman R and Aguinaga M (2013) The p.L750V mutation in the NLRP7 gene is frequent in Mexican patients with recurrent molar pregnancies and is not associated with recurrent pregnancy loss. Prenatal Diagnosis 33 (3): 205-208.

Fallahian M, Sebire NJ, Savage PM, Seckl MJ and Fisher RA (2013) Mutations in NLRP7 and KHDC3L confer a complete hydatidiform mole phenotype on digynic triploid conceptions. Human Mutation 34 (2): 301-308.

Fisher RA, Lavery SA, Carby A, et al. (2011) What a difference an egg makes. Lancet 378 (9807): 1974.

Grimes DA (1984) Epidemiology of gestational trophoblastic disease. American Journal of Obstetrics and Gynecology 150 (3): 309-318.

Hayward BE, De Vos M, Talati N, et al. (2009) Genetic and epigenetic analysis of recurrent hydatidiform mole. Human Mutation 30 (5): E629-E639.

Helwani MN, Seoud M, Zahed L, et al. (1999) A familial case of recurrent hydatidiform molar pregnancies with biparental genomic contribution. Human Genetics 105 (1-2): 112-115. 
Ho PC, Mak LW, Lawton JW and Ma HK (1980) Immunological parameters in gestational trophoblastic neoplasia. Journal of Reproductive Immunology 1 (5-6): 307-319.

Hou JL, Wan XR, Xiang Y, Qi QW and Yang XY (2008) Changes of clinical features in hydatidiform mole: analysis of 113 cases. Journal of Reproductive Medicine 53 (8): 629-633.

Ito Y, Maehara K, Kaneki E, et al. (2015) Novel nonsense mutation in the NLRP7 gene associated with recurrent hydatidiform mole. Gynecologic and Obstetric Investigation 81 (4): 353-358.

Joshi S, Davies H, Sims LP, Levy SE and Dean J (2007) Ovarian gene expression in the absence of FIGLA, an oocyte-specific transcription factor. BMC Developmental Biology 7: 67.

Judson H, Hayward BE, Sheridan E and Bonthron DT (2002) A global disorder of imprinting in the human female germ line. Nature 416 (6880): 539-542.

Khanna A, Khanna S, Gupta RM, Gupta S and Khanna AK (1985) Immunological status of trophoblastic neoplasia. Journal of Surgical Oncology 28 (1): 4-6.

Khare S, Dorfleutner A, Bryan NB, et al. (2012) An NLRP7-containing inflammasome mediates recognition of microbial lipopeptides in human macrophages. Immunity 36 (3): 464-476.

Kim JH, Park DC, Bae SN, Namkoong SE and Kim SJ (1998) Subsequent reproductive experience after treatment for gestational trophoblastic disease. Gynecologic Oncology 71 (1): 108-112.

Kinoshita T, Wang Y, Hasegawa M, Imamura R and Suda T (2005) PYPAF3, a PYRIN-containing APAF-1-like protein, is a feedback regulator of caspase-1-dependent Interleukin-1\{beta\} secretion. Journal of Biological Chemistry 280 (23): 21720-21725.

Kou YC, Shao L, Peng HH, et al. (2008) A recurrent intragenic genomic duplication, other novel mutations in NLRP7 and imprinting defects in recurrent biparental hydatidiform moles. Molecular Human Reproduction 14 (1): 33-40.

Kronfol NM, Iliya FA and Hajj SN (1969) Recurrent hydatidiform mole: a report of five cases with review of the literature. Le Journal Médical Libanais 22 (4): 507-520.

Mahadevan S, Wen S, Wan YW, et al. (2014) NLRP7 affects trophoblast lineage differentiation, binds to overexpressed YY1 and alters $\mathrm{CpG}$ methylation. Human Molecular Genetics 23 (3): 706-716.

Mangili G, Garavaglia E, Cavoretto P, et al. (2008) Clinical presentation of hydatidiform mole in northern Italy: has it changed in the last 20 years? American Journal of Obstetrics and Gynecology 198 (3, 302): e301-e304.

Messaed C, Akoury E, Djuric U, et al. (2011a) NLRP7, a nucleotide oligomerization domain-like receptor protein, is required for normal cytokine secretion and co-localizes with Golgi and the microtubule-organizing center. Journal of Biological Chemistry 286 (50): 43313-43323.

Messaed C, Chebaro W, Di Roberto RB, et al. (2011b) NLRP7 in the spectrum of reproductive wastage: rare non-synonymous variants confer genetic susceptibility to recurrent reproductive wastage. Journal of Medical Genetics 48 (8): 540-548.

Moglabey YB, Kircheisen R, Seoud M, et al. (1999) Genetic mapping of a maternal locus responsible for familial hydatidiform moles. Human Molecular Genetics 8 (4): 667-671.

Murdoch S, Djuric U, Mazhar B, et al. (2006) Mutations in NALP7 cause recurrent hydatidiform moles and reproductive wastage in humans. Nature Genetics 38 (3): 300-302.

Nguyen NM, Zhang L, Reddy R, et al. (2014) Comprehensive genotype-phenotype correlations between NLRP7 mutations and the balance between embryonic tissue differentiation and trophoblastic proliferation. Journal of Medical Genetics 51 (9): 623-634.

Ohsugi M, Zheng P, Baibakov B, Li L and Dean J (2008) Maternally derived FILIA-MATER complex localizes asymmetrically in cleavage-stage mouse embryos. Development 135 (2): 259-269.

Osathanondh R, Berkowitz RS, de Cholnoky C, et al. (1986) Hormonal measurements in patients with theca lutein cysts and gestational trophoblastic disease. Journal of Reproductive Medicine 31 (3): 179-183.

Parry DA, Logan CV, Hayward BE, et al. (2011) Mutations causing familial biparental hydatidiform mole implicate c6orf221 as a possible regulator of genomic imprinting in the human oocyte. American Journal of Human Genetics 89 (3): 451-458.

Qian J, Cheng Q, Murdoch S, et al. (2011) The genetics of recurrent hydatidiform moles in China: correlations between NLRP7 mutations, molar genotypes, and reproductive outcomes. Molecular Human Reproduction 17 (10): 612-619.

Ramsey PS, Van Winter JT, Gaffey TA and Ramin KD (1998) Eclampsia complicating hydatidiform molar pregnancy with a coexisting, viable fetus. A case report. Journal of Reproductive Medicine 43 (5): 456-458.

Reddy R, Akoury E, Phuong Nguyen NM, et al. (2013) Report of four new patients with protein-truncating mutations in C6orf221/KHDC3L and colocalization with NLRP7. European Journal of Human Genetics 21 (9): 957-964.

Reddy R, Nguyen NM, Sarrabay G, et al. (2016) The genomic architecture of NLRP7 is Alu rich and predisposes to disease-associated large deletions. European Journal of Human Genetics 24 (10): 1445-1452.

Rezaei M, Nguyen NMP, Foroughinia L, et al. (2016) Two novel mutations in the KHDC3L gene in Asian patients with recurrent hydatidiform mole. Human Genome Variation 3: 16027.

Sanchez-Delgado M, Martin-Trujillo A, Tayama C, et al. (2015) Absence of maternal methylation in biparental hydatidiform moles from women with NLRP7 maternal-effect mutations reveals widespread placenta-specific imprinting. PLoS Genetics 11 (11): e1005644.

Sebire NJ, Rees H, Paradinas F, Seckl M and Newlands E (2001) The diagnostic implications of routine ultrasound examination in histologically confirmed early molar pregnancies. Ultrasound in Obstetrics and Gynecology 18 (6): 662-665.

Sebire NJ, Fisher RA, Foskett M, et al. (2003) Risk of recurrent hydatidiform mole and subsequent pregnancy outcome following complete or partial hydatidiform molar pregnancy. BJOG: An International Journal of Obstetrics and Gynaecology 110 (1): 22-26.

Sebire NJ, Savage PM, Seckl MJ and Fisher RA (2013) Histopathological features of biparental complete hydatidiform moles in women with NLRP7 mutations. Placenta 34 (1): 50-56.

Singer H, Biswas A, Zimmer N, et al. (2014) NLRP7 inter-domain interactions: the NACHT-associated domain is the physical mediator for oligomeric assembly. Molecular Human Reproduction 20 (10): 990-1001.

Slim R, Bagga R, Chebaro W, Srinivasan R and Agarwal N (2009) A strong founder effect for two NLRP7 mutations in the Indian population: an intriguing observation. Clinical Genetics 76 (3): 292-295.

Slim R, Ao A, Surti U, et al. (2011) Recurrent triploid and dispermic conceptions in patients with NLRP7 mutations. Placenta 32 (5): 409-412. 
Slim R, Coullin P, Diatta AL, et al. (2012) NLRP7 and the genetics of post-molar choriocarcinomas in Senegal. Molecular Human Reproduction 18 (1): 52-56.

Sunde L, Vejerslev LO, Jensen MP, et al. (1993) Genetic analysis of repeated, biparental, diploid, hydatidiform moles. Cancer Genet Cytogenet, 66 (1): 16-22.

Szulman AE and Surti U (1978) The syndromes of hydatidiform mole. II. Morphologic evolution of the complete and partial mole. American Journal of Obstetrics and Gynecology 132 (1): 20-27.

Tian X, Pascal G and Monget P (2009) Evolution and functional divergence of NLRP genes in mammalian reproductive systems. BMC Evolutionary Biology 9: 202.

Tomoda Y, Fuma M, Saiki N, Ishizuka N and Akaza T (1976) Immunologic studies in patients with trophoblastic neoplasia. American Journal of Obstetrics and Gynecology 126 (6): 661-667.

Ulker V, Gurkan H, Tozkir H, et al. (2013) Novel NLRP7 mutations in familial recurrent hydatidiform mole: are NLRP7 mutations a risk for recurrent reproductive wastage? European Journal of Obstetrics, Gynecology, and Reproductive Biology 170 (1): 188-192.

Vejerslev LO, Sunde L, Hansen BF, et al. (1991) Hydatidiform mole and fetus with normal karyotype: support of a separate entity. Obstetrics and Gynecology 77 (6): 868-874.

Zhao J, Moss J, Sebire NJ, et al. (2006) Analysis of the chromosomal region 19q13.4 in two Chinese families with recurrent hydatidiform mole. Human Reproduction 21 (2): 536-541.

Zheng P and Dean J (2009) Role of Filia, a maternal effect gene, in maintaining euploidy during cleavage-stage mouse embryogenesis. Proceedings of the National Academy of Sciences 106 (18): 7473-7478.

Zhou Y, Shah SZ, Yang L, et al. (2016) Virulent mycobacterium bovis Beijing strain activates the NLRP7 inflammasome in THP-1 macrophages. PLoS One 11 (4): e0152853.

\section{Further Reading}

Altieri A, Franceschi S, Ferlay J, Smith J and La Vecchia C (2003) Epidemiology and aetiology of gestational trophoblastic diseases. Lancet Oncology 4 (11): 670-678.

Banet N, DeScipio C, Murphy KM, et al. (2014) Characteristics of hydatidiform moles: analysis of a prospective series with p57 immunohistochemistry and molecular genotyping. Modern Pathology 27 (2): 238-254.

Berkowitz RS and Goldstein DP (2009) Clinical practice. Molar pregnancy. New England Journal of Medicine 360 (16): 1639-1645.

Choufani S, Shapiro JS, Susiarjo M, et al. (2011) A novel approach identifies new differentially methylated regions (DMRs) associated with imprinted genes. Genome Research 21 (3): 465-476.

Li HW, Tsao SW and Cheung AN (2002) Current understandings of the molecular genetics of gestational trophoblastic diseases. Placenta 23 (1): 20-31.

Sauthier P, Breguet M, Rozenholc A and Sauthier M (2015) Quebec trophoblastic disease registry: how to make an easy-to-use dynamic database. International Journal of Gynecological Cancer 25 (4): 729-733.

Seckl MJ, Sebire NJ and Berkowitz RS (2010) Gestational trophoblastic disease. Lancet 376 (9742): 717-729.

Sundvall L, Lund H, Niemann I, et al. (2013) Tetraploidy in hydatidiform moles. Human Reproduction 28 (7): 2010-2020.

Surti U, Hoffner L, Kolthoff M, et al. (2006) Persistent gestational trophoblastic disease after an androgenetic/biparental fetal chimera: a case report and review. International Journal of Gynecological Pathology 25 (4): 366-372. 